05,03

\title{
Конкуренция зонного и прыжкового механизмов переноса носителей заряда в тонких пленках $\mathrm{Ge}: \mathrm{Mn}$
}

\author{
(ㄱ А.И. Дмитриев, Л.И. Буравов \\ Институт проблем химической фризики РАН, \\ Черноголовка, Россия \\ E-mail: aid@icp.ac.ru
}

(Поступила в Редакцию 2 августа 2016 г.)

Исследованы закономерности дырочного транспорта в его взаимосвязи с перколяционным магнетизмом, обусловленным локализованными носителями заряда, одновременно участвующими в формировании магнитных и электрических свойств тонких пленок $\mathrm{Ge}: \mathrm{Mn}$. Установлено, что при температурах $T>22 \mathrm{~K}$ в образцах $\mathrm{Ge}: \mathrm{Mn}(2$ at.\% Mn) происходит безактивационный зонный перенос носителей заряда. При низких температурах имеет место прыжковый механизм с переменной длиной прыжка.

Работа поддержана грантом Президента РФ МК-5754.2016.3.

DOI: 10.21883/FTT.2017.03.44163.317

\section{1. Введение}

Одним из ключевых направлений спинтроники, да и физики твердого тела в целом, является создание и управление спиновой поляризацией носителей заряда в полупроводниках. В настоящее время надежно установлено, что в самых различных полупроводниках, сильно легированных переходными металлами (1-10 at.\%), при низких температурах наблюдается дальний магнитный порядок за счет косвенного обменного взаимодействия, передаваемого носителями заряда [1-4]. Поэтому разбавленные магнитные полупроводники - эффективная среда для создания спиновой поляризации носителей заряда.

Известные трудности в изготовлении разбавленных магнитных полупроводников и интерпретации их магнитных свойств обусловлены плохой растворимостью примеси переходных металлов в полупроводниковой матрице и, как следствие, образованием ферромагнитных кластеров вторичной фазы - наночастиц сплава легирующей примеси и полупроводника [5-10]. При этом разбавленные магнитные полупроводники имеют две магнитные подсистемы: твердый раствор ионов переходных металлов, являющихся, как правило, примесью замещения, и кластеры вторичной фазы. Присутствие этих кластеров обычно считалось вредным, так как кластеризация примеси приводит к обеднению подсистемы диспергированных ионов переходных металлов, а значит к понижению соответствующей температуры Кюри, которая и без того обычно не превышает $110 \mathrm{~K}$.

Недавно появились экспериментальные свидетельства того, что в разбавленном магнитном полупроводнике $\mathrm{GaSb}: \mathrm{Mn}$ спиновая поляризация носителей заряда может быть чувствительна к намагниченности кластеров $\mathrm{MnSb}$ [10]. И это далеко не единственный пример. В настоящее время различными группами исследователей накоплен экспериментальный материал, свидетельствующий о спин-поляризованном состоянии носителей заряда в разбавленных магнитных полупроводниках, содержащих ферромагнитные кластеры вторичной фазы. В качестве примеров можно указать: магнитный круговой дихроизм и фарадеевское вращение в пленках GaAs:Mn c кластерами MnAs, полученных методом молекулярно-лучевой эпитаксии [5,6]; положительное магнетосопротивление в пленках GaAs:Mn с кластерами MnAs, полученных методом ионной имплантации [7]; анизотропное микроволновое магнетосопротивление в пленках $\mathrm{Ge}: \mathrm{Mn}$ с кластерами $\mathrm{Mn}_{5} \mathrm{Ge}_{3}$, полученных методом ионной имплантации [8,9]; аномальный эффект Холла и анизотропное магнетосопротивление в пленках $\mathrm{GaSb}: \mathrm{Mn}$ с кластерами $\mathrm{MnSb}$, полученных методом импульсного лазерного осаждения [10]. Заметим, что перечисленные выше „спинтронические“ эффекты наблюдаются при комнатных температурах в отсутствие дальнего магнитного порядка в образце. Более того эти эффекты наблюдаются в самых разных материалах, приготовленных различными методами. Возникает совершенно новый подход к созданию и управлению спиновой поляризацией носителей заряда в разбавленных магнитных полупроводниках с помощью ферромагнитных кластеров, внедренных в полупроводник.

Ранее нами были исследованы тонкие пленки $\mathrm{Ge}: \mathrm{Mn}$, полученные методом ионной имплантации $[11,12]$. В образцах наблюдалось две температуры Кюри. Высокая температура Кюри $\sim 300 \mathrm{~K}$ отвечает кластерам $\mathrm{Mn}_{5} \mathrm{Ge}_{3}$. Ферромагнитное упорядочение в подсистеме диспергированных ионов, происходящее по перколяционному сценарию, наблюдалось при температурах $\sim 10 \mathrm{~K}$. В настоящей работе созданы условия для исследования фундаментальных закономерностей электропроводности и ее взаимосвязи с перколяционным магнетизмом, обусловленным носителями заряда, одновременно участвующих в формировании магнитных и электрических свойств. Исследование транспортных явлений в системах с перколяционным ферромагнетизмом является 
новой фундаментальной задачей. Целью настоящей работы являлось установление механизма переноса носителей заряда и закономерностей дырочного транспорта в перколяционно намагниченных тонких пленках $\mathrm{Ge}: \mathrm{Mn}$, содержащих кластеры $\mathrm{Mn}_{5} \mathrm{Ge}_{3}$.

\section{2. Методика}

Образцы Ge:Mn (2at.\% Mn) толщиной 120 nm были изготовлены методом ионной имплантации ионов марганца в пластины монокристаллического германия (рис. 1). Подробно методика изготовления образцов тонких пленок описана в работах [13-16]. Структура пленок и валентное состояние ионов марганца были исследованы ранее методами просвечивающей электронной микроскопии, рентгеновской дифракции, рентгеновской фотоэлектронной спектроскопии [13-16]. Было установлено, что пленки $\mathrm{Ge}: \mathrm{Mn}$ содержат кластеры $\mathrm{Ge}_{3} \mathrm{Mn}_{5}$ со средним диаметром $4.3 \mathrm{~nm}$, а также изолированные ионы $\mathrm{Mn}^{2+}$ в узлах кристаллической решетки германия (рис. 1).

Измерения магнитного момента пленок проводили с помощью СКВИД магнитометра MPMS 5XL, Quantum Design при температурах $T=2-350 \mathrm{~K}$ в постоянном магнитном поле напряженностью $H=1 \mathrm{kOe}$.

Электрическое сопротивление образцов на постоянном токе измеряли стандартным четерехконтактным способом при температурах $T=4.2-300 \mathrm{~K}$. Образцы с характерными размерами $\sim 5 \mathrm{~mm}$ приклеивали токопроводящей графитой пастой к четырем тонким платиновым проволокам, укрепленным на измерительном модуле. Модуль с приклеенным образцом монтировали внутри медного стаканчика на конце тонкостенной трубки из

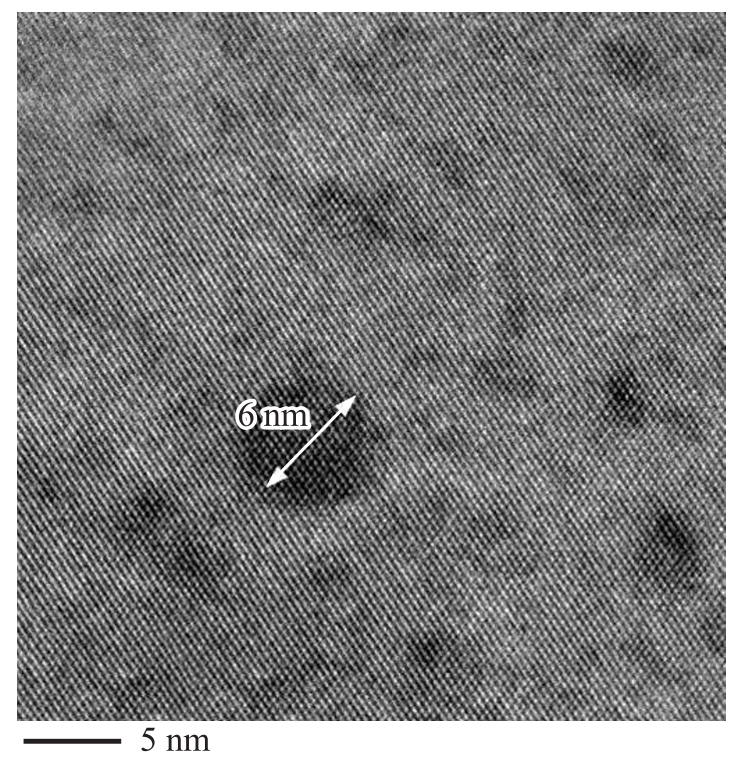

Рис. 1. Изображение поперечного сечения ионноимплантированной пленки $\mathrm{Ge}: \mathrm{Mn}(2 \mathrm{at} . \% \mathrm{Mn})$, полученное на просвечивающем электронном микроскопе. нержавеющей стали, которую с помощью электродвигателя РД-09, снабженного редуктором оборотов, плавно погружали в транспортный гелиевый дьюар. Скорость изменения темпертуры в среднем составляла $\sim 1 \mathrm{~K} / \mathrm{min}$. В качестве источника тока использовался программируемый источник напряжения Hewlett-Packard 59501B Power Supply Programmer с большим балластным сопротивлением $\sim 400 \mathrm{kOhm}$. Измерения были выполнены на электрическом токе $25 \mu \mathrm{A}$. Напряжение на образце регистрировалось с помощью нановольтметра Keithely 181 Nanovoltmeter.

\section{3. Результаты и обсуждение}

3.1. Магнитные свойства. Магнитные свойства тонких пленок Ge:Mn c различной концентрацией марганца, выращенных при различных температурах, подробно были исследованы нами ранее в работах $[11,12]$. Здесь кратко приведены лишь те экспериментальные результаты, которые необходимы для обсуждения закономерностей электронного транспорта образцов во взаимосвязи с их магнитными свойствами. На рис. 2, $a$ приведена температурная зависимость магнитного момента $M(T)$ образца Ge: $\mathrm{Mn}$ (2 at.\% Mn). При температуpax выше $T=266 \mathrm{~K}$ образец находится в парамагнитном состоянии, а зависимость $M(T)$ описывается законом Кюри-Вейса (рис. 2,a). Понижение температуры приводит к ферромагнитному упорядочению в подсистеме кластеров $\mathrm{Ge}_{3} \mathrm{Mn}_{5}$. Температурная зависимость магнитного момента в интервале температур $T=30-230 \mathrm{~K}$ описывается законом Блоха (рис. 2,a). При самых низких температурах $T<10 \mathrm{~K}$ в образце происходит второй магнитный фазовый переход (рис. 2,a), связанный с дальним магнитным упорядочением в подсистеме диспергированных ионов $\mathrm{Mn}^{2+}$. При этом вид температурной зависимости $M(T)$ становится иным (рис. 2,a). Ранее нами было установлено, что в температурном интервале $T=2-10 \mathrm{~K}$ зависимость $M(T)$ описывается формулой, полученной в рамках теории протекания $[11,12]$. В основе перколяционной модели лежит предположение о том, что обменное взаимодействие обусловлено локализованными носителями заряда (в случае акцепторной примеси марганца - дырками). Для корректного анализа магнитных свойств подсистемы диспергированных ионов $\mathrm{Mn}^{2+}$ необходимо предварительно выделить ее вклад в суммарный магнитный момент. Для этого в интервале температур $T=30-230 \mathrm{~K}$, где ферромагнетизм образца обусловлен только вкладом кластеров $\mathrm{Ge}_{3} \mathrm{Mn}_{5}$, а изолированные ионы $\mathrm{Mn}^{2+}$ находятся в парамагнитном состоянии, была выполнена аппроксимация зависимости $M(T)$ законом Блоха (рис. 2,a). Затем кривая Блоха была экстраполирована в область низких температур и вычтена из суммарного магнитного момента (рис. 2,a). Результат описанной процедуры представлен на рис. 2, $b$. Сплошной линией 

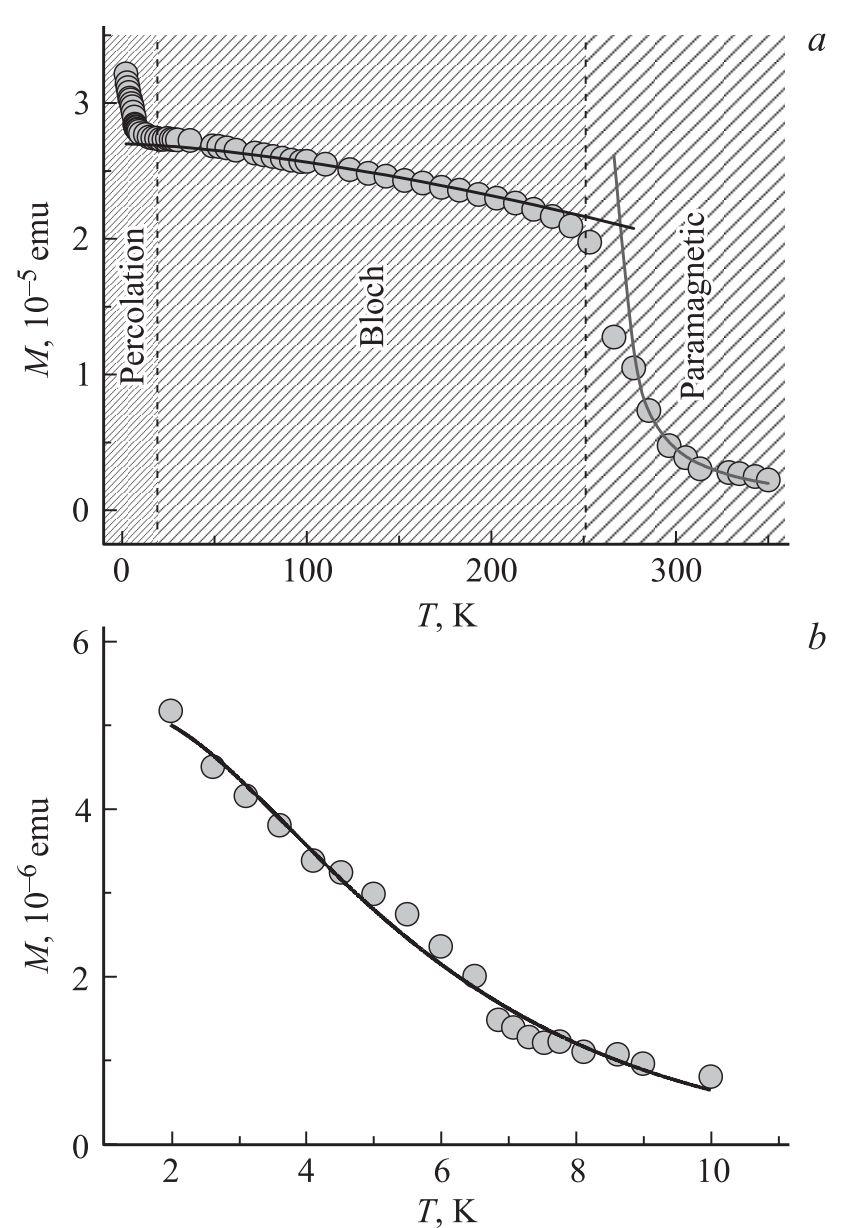

Рис. 2. a) Температурная зависимость магнитного момента $M(T)$ образца $\mathrm{Ge}: \mathrm{Mn}(2 \mathrm{at} . \% \mathrm{Mn})$. Температурный интервал разбит на три области, различающиеся магнитным состоянием образца: парамагнетик, блоховский ферромагнетик, перколяционный ферромагнетик. Серой линией показана аппроксимация высокотемпературной части зависимости $M(T)$ формулой Кюри-Вейса. Черной линией показана аппроксимация зависимости $M(T)$ при температурах $T=30-230 \mathrm{~K}$ формулой Блоха и экстраполяция блоховской кривой в область низких температур. $b$ ) Низкотемпературный фрагмент зависимости $M(T)$ подсистемы диспергированных ионов $\mathrm{Mn}^{2+}$ и ее аппроксимация формулой, полученной в рамках теории протекания.

показана аппроксимация выражением [17]

$$
M(T)=M(0)\left[1-\exp \left(-L^{3} n_{h} \ln ^{3} \frac{S J}{T}\right)\right],
$$

где $M(0)$ - магнитный момент при $0 \mathrm{~K}, L-$ радиус локализации дырок, $n_{h}=2.4 \cdot 10^{20} \mathrm{~cm}^{-3}-$ концентрация дырок $[11,12], S=5 / 2-$ спин ионов $\mathrm{Mn}^{2+}, J-$ обменный интеграл, $T$ - температура. Из аппроксимации были определены значения обменного интеграла $J=66 \mathrm{~K}$, и радиус локализации дырок $L=6 \AA[11,12]$.

3.2. Электронно-транспортные свойства. На рис. 3 приведена температурная зависимость электрического сопротивления $\mathrm{R}(\mathrm{T})$ образца $\mathrm{Ge}: \mathrm{Mn}$ (2 at.\% Mn), нормированного на его значение при $300 \mathrm{~K}$. При температурах $T>22 \mathrm{~K}$ зависимость $R(T)$ носит металлический характер, т.к. в этой области температур $d R / d T>0$. Напротив, при температурах ниже $22 \mathrm{~K}$ производная $d R / d T<0$, что соответствует полупроводящему состоянию образца.

Высокотемпературный участок кривой $R(T)$ аппроксимирован степенной функцией $R \sim T^{\alpha}, \alpha=1.56$ (рис. 3). Значение показателя степени $\alpha$, определенное из аппроксимации, близко к значению $3 / 2$, соответствующему рассеянию дырок на акустических фононах $[18,19]$. В условиях сильного легирования германия акцепторной примесью марганца уровень Ферми попадает в валентную зону (или находится на расстоянии не более $k_{\mathrm{B}} T$ от ее границы), что обеспечивает „свободное“ движение дырок без термической активации. Полученный результат означает, что в тонких пленках $\mathrm{Ge}: \mathrm{Mn}$ (2 at.\% Mn) при температурах $T>22 \mathrm{~K}$ имеет место безактивационный зонный перенос носителей заряда.

Низкотемпературному участку кривой $R(T)$ может соответствовать несколько механизмов переноса носителей заряда: активационный механизм переноса термически делокализованных носителей; прыжки локализованных носителей на ближайшие центры; прыжки с переменной длиной по Мотту или по ЭфросуШкловскому [18]. Наша задача установить, какой из механизмов переноса носителей заряда реализуется в образце при низких температурах.

Попытка аппроксимировать температурную зависимость $R(T)$ функцией $R \sim \exp \left(E_{0} / T\right)$ приводит к некорректному значению уровня примеси $E_{0}=6.8 \mathrm{meV}$, более чем на порядок меньшему известных значений для марганца $0.16 \mathrm{eV}$ и $0.37 \mathrm{eV}$ [20]. Также непригодной является модель прыжков локализованных носителей на ближай-

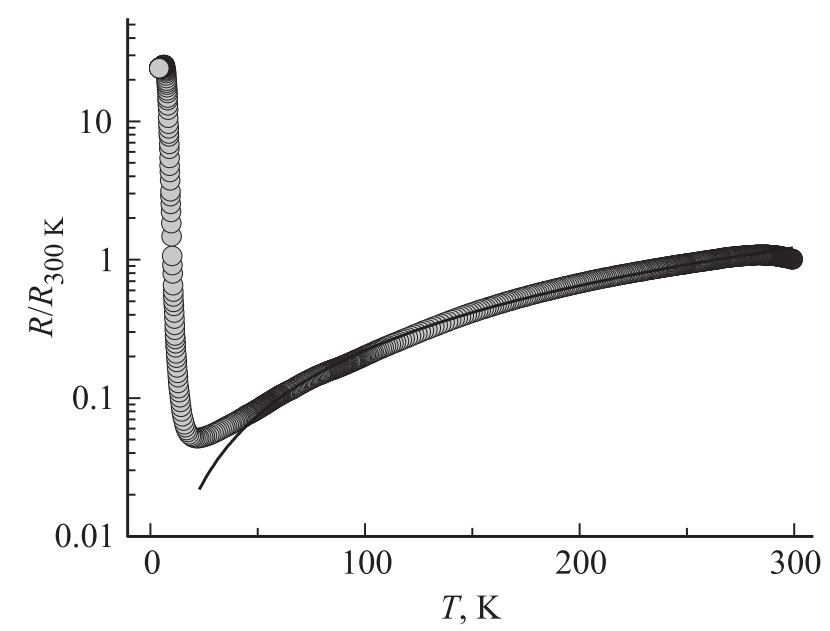

Рис. 3. Температурная зависимость электрического сопротивления $R(T)$ образца $\mathrm{Ge}: \mathrm{Mn}$ (2 at.\% $\mathrm{Mn}$ ), нормированного на его значение при $300 \mathrm{~K}$. Сплошной линией показана аппроксимация высокотемпературной части $(T>22 \mathrm{~K})$ кривой $R(T)$ степенной функцией. 
шие центры, когда $R \sim \exp \left(\left(\mu-E_{0}\right) / T\right)$. Здесь $\mu-$ уровень Ферми, разность $\mu-E_{0}-$ энергия активации прыжковой проводимости. В этом случае энергия активации $\mu-E_{0}=6.8 \mathrm{meV}$. Последнее равенство означает, что уровень Ферми лежит вблизи примесных уровней марганца $0.16 \mathrm{eV}$ и $0.37 \mathrm{eV}$ от потолка валентной зоны. Это противоречит высокотемпературным экспериментальным результатам, согласно которым уровень Ферми находится на расстоянии не более $k_{\mathrm{B}} T$ от потолка валентной зоны. Ввиду того, что оба рассмотренных механизма неприменимы для описания температурных зависимостей электрического сопротивления образца $\mathrm{Ge}: \mathrm{Mn}(2$ at.\% Mn), соответствующие аппроксимации кривых $R(T)$ в статье не приводятся.

На рис. $4, a$ и рис. $4, b$ приведен низкотемпературный фрагмент зависимости $R(T)$ и ее аппроксимация методом наименьших квадратов формулами Мотта (рис. $4, a$ ) и Эфроса-Шкловского (рис. 4, $b$ ). В обоих случаях коэффициент детерминации был не меньше $R^{2}=0.96$.

В модели Мотта электрическое сопротивление изменяется с температурой по закону [18]

$$
R=R_{0} \exp \left(\frac{T_{M}}{T}\right)^{\frac{1}{4}}
$$

где $R_{0}$ - предэкспоненциальный фактор, считающийся независимым от температуры; эффективная температуpa $T_{M}$ - характерная величина с размерностью температуры, зависящая от плотности состояний в окрестности уровня Ферми, и определяющая среднюю длину прыжка. Определенная из аппроксимации величина эффективной температуры составляет $T_{M}=1.8 \cdot 10^{7} \mathrm{~K}$. Обычно в самых разных материалах эффективная температура изменяется в пределах $T_{M}=10^{3}-10^{5}[21]$, однако в наноструктурах, в частности, в квантовых точках $\mathrm{CdSe}$ величина $T_{M}$ достигает значения $10^{8} \mathrm{~K}$ [22]. В литературе не встречается значений $T_{M}$ для материалов, сходных с исследуемыми здесь тонкими пленками $\mathrm{Ge}: \mathrm{Mn}$ (2 at.\% Mn), поэтому для оценки применимости закона Мотта и справедливости определенного из него значения $T_{M}$ было сделано следующее. Эффективная температура $T_{M}$ была пересчитана в радиус локализации дырок, и полученное таким образом значение $L$ было сравнено с соответствующим значением, определенным из анализа магнитных данных. В законе Мотта температура $T_{M}$ определяется формулой [22]

$$
T_{M}=\frac{21.2}{k_{\mathrm{B}} g L^{3}},
$$

где $g=3 n_{h} / \mu=2.2 \cdot 10^{21} \mathrm{eV}^{-1} \cdot \mathrm{cm}^{-3}$ - плотность состояний в окрестности уровня Ферми. Из выражения (4) было оценено значение радиуса локализации дырок $L=2 \AA$, которое оказалось близко к соответствующему значению $6 \AA$, определенному выше из анализа магнитных данных. Близкие значения радиуса локализации дырок, определенные различными методами, с одной стороны подтверждают применимость закона Мотта и
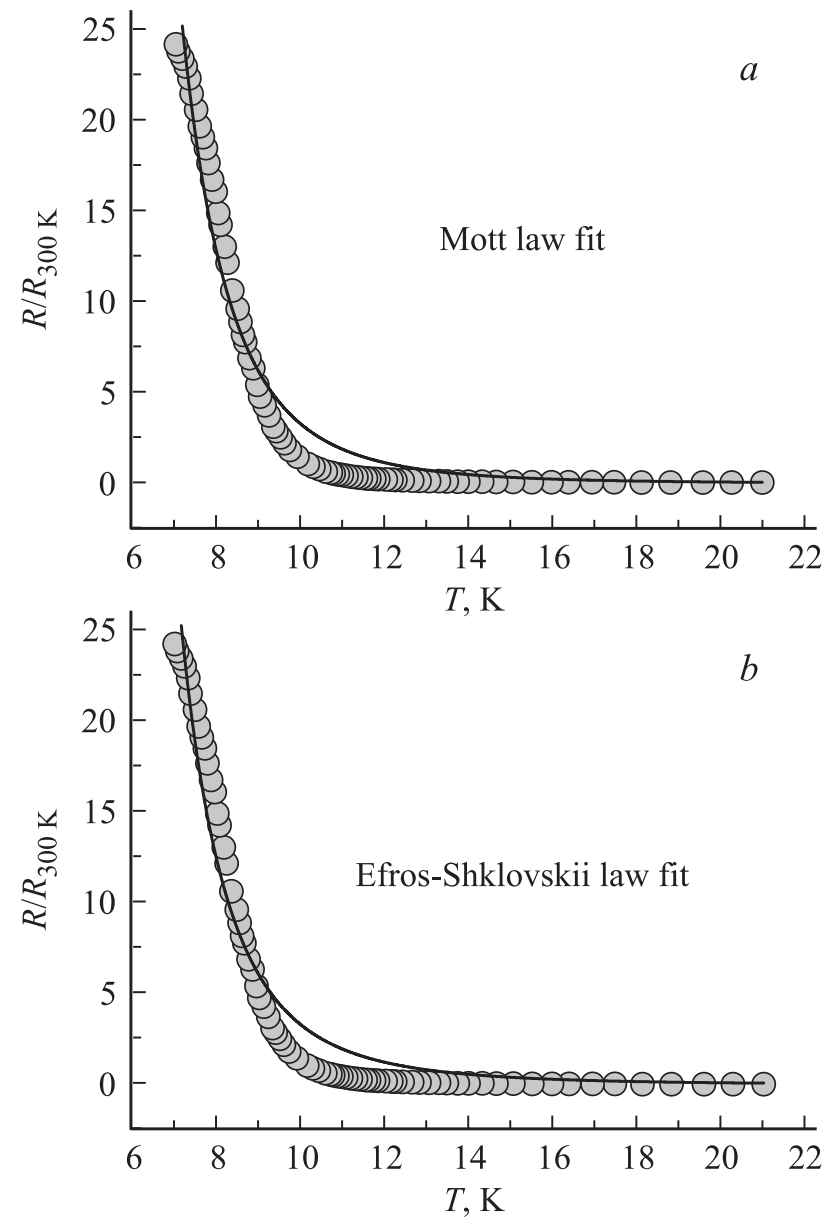

Рис. 4. Низкотемпературный фрагмент зависимости $R(T)$ и ее аппроксимация формулами Мотта $(a)$ и ЭфросаШкловского $(b)$.

справедливость определенного значения $T_{M}$, с другой являются еще одним подтверждением перколяционного сценария ферромагнитного упорядочения в тонких пленках Ge:Mn.

В модели Эфроса-Шкловского зависимость $R(T)$ описывается формулой [18]

$$
R=R_{0} \exp \left(\frac{T_{E S}}{T}\right)^{\frac{1}{2}}
$$

где $R_{0}$ - коэффициент, зависимостью от температуры которого обычно пренебрегают; $T_{\mathrm{ES}}-$ величина с размерностью температуры, определяемая минимумом показателя экспоненты для величины сопротивления в подсетке Абрахамса-Миллера [18]. Определенная из аппроксимации величина эффективной температуры составляет $T_{\mathrm{ES}}=3.1 \cdot 10^{3} \mathrm{~K}$. Величина $T_{\mathrm{ES}}$ обычно изменяется в пределах от $1 \mathrm{~K}$ до $500 \mathrm{~K}$ [21], однако в наноструктурах, например, в квантовых точках CdSe $T_{\mathrm{ES}}$ достигает значения $10^{4} \mathrm{~K}$ [22]. Нам не удалось найти в литературе значений $T_{\mathrm{ES}}$ для материалов, сходных с исследуемыми здесь тонкими пленками $\mathrm{Ge}: \mathrm{Mn}$ (2 at.\% Mn), поэтому 
для оценки применимости закона Эфроса-Шкловского и справедливости определенного из него значения $T_{\mathrm{ES}}$ была выполнена процедура, аналогичная той, что описана выше. В законе Эфроса-Шкловского температура $T_{\mathrm{ES}}$ определяется формулой [22]

$$
T_{\mathrm{ES}}=\frac{2.8 e^{2}}{\chi k_{\mathrm{B}} L},
$$

где $\chi=16$ - диэлектрическая проницаемость германия, $e-$ элементарный электрический заряд. Используя формулу (5) была выполнена оценка значения радиуса локализации дырок $L=8 \AA$, которое оказалось близко к значению $6 \AA$, полученному из анализа магнитных данных.

Оба значения величины $L$, полученные в рамках моделей Мотта и Эфроса-Шкловского, одинаково близки к ,референсному“ значению $L=6 \AA$. Это затрудняет выбор в пользу того или иного механизма переноса носителей заряда, реализующегося в образце при низких температурах. Известно, что различать прыжки с переменной длиной прыжка по Мотту или по Эфросу-Шкловскому иногда бывает очень трудно [18]. Для этого существуют специальные приемы, описанные в работе [18]. Как минимум для этого уже нельзя считать температурно-независимыми предэкспоненциальные факторы $R_{0}$. Эта работа выходит за рамки настоящей статьи и требует отдельного обсуждения.

\section{4. Выводы}

Изучены механизмы переноса носителей заряда в перколяционно намагниченных тонких пленках $\mathrm{Ge}: \mathrm{Mn}$. Установлено, что электронно-транспортные свойства образцов $\mathrm{Ge}: \mathrm{Mn}$ (2 at.\% Mn) обусловлены конкуренцией зонного и прыжкового механизмов. При высоких темпеpaтурах $(T>22 \mathrm{~K})$ доминирующим механизмом является безактивационный зонный перенос дырок с рассеянием на акустических фононах. При низких температурах имеет место прыжковая проводимость с переменной длиной прыжка. Значения радиуса локализации дырок, определенные в настоящей работе из данных по электропроводности, и полученные ранее из магнитных данных, оказались близки. Что является подтверждением перколяционного сценария ферромагнитного упорядочения в тонких пленках $\mathrm{Ge}: \mathrm{Mn}$.

Авторы признательны О.Л. Казаковой за образцы и данные просвечивающей электронной микроскопии.

\section{Список литературы}

[1] J.K. Furdyna, N. Samarth. J. Appl. Phys. 61, 3526 (1987).

[2] T. Dietl, H. Ohno, F. Matsukura, J. Cibert, D. Ferrand. Science 287, 1019 (2000).

[3] T. Jungwirth, J. Sinova, J. Mašek, J. Kučera, A.H. MacDonald. Rev. Mod. Phys. 78, 809 (2006).
[4] S. Kuroda, N. Nishizawa, K. Takita, M. Mitome, Y. Bando, K. Osuch, T. Dietl. Nature Mater. 6, 440 (2007).

[5] H. Akinaga, S. Miyanishi, K. Tanaka, W. Van Roy, K. Onodera. Appl. Phys. Lett. 76, 97 (2000).

[6] H. Shimizu, M. Tanaka. J. Appl. Phys. 89, 7281 (2001).

[7] Sh.U. Yuldashev, Y. Shon, Y.H. Kwon, D.J. Fu, D.Y. Kim, H.J. Kim, T.W. Kang, X. Fan. J. Appl. Phys. 90, 3004 (2001).

[8] Р.Б. Моргунов, А.И. Дмитриев. ФТТ 51, 1873 (2009).

[9] А.И. Дмитриев, Р.Б. Моргунов, О.Л. Казакова. ФТТ 44, 320 (2010).

[10] В.В. Рыльков, Б.А. Аронзон, Ю.А. Данилов, Ю.Н. Дроздов, В.П. Лесников, К.И. Маслаков, В.В. Подольский. ЖЭТФ 127, 838 (2005).

[11] R.B. Morgunov, A.I. Dmitriev, O.L. Kazakova. Phys. Rev. B 80, 085205 (2009).

[12] А.И. Дмитриев, Р.Б. Моргунов, О.Л. Казакова, Й. Танимото. ЖЭТФ 135, 1134 (2009).

[13] L. Ottaviano, M. Passacantando, A. Verna, R. Gunnella, E. Principi, A. Di Cicco, G. Impellizzeri, F. Priolo. J. Appl. Phys. 100, 063528 (2006).

[14] L. Ottaviano, M. Passacantando, S. Picozzi, A. Continenza, R. Gunnella, A. Verna, G. Bihlmayer, G. Impellizzeri, F. Priolo. Appl. Phys. Lett. 88, 061907 (2006).

[15] L. Ottaviano, M. Passacantando, A. Verna, F. D’Amico, R. Gunnella. Appl. Phys. Lett. 90, 242105 (2007).

[16] M. Passacantando, L. Ottaviano, F. D’Orazio, F. Lucari, M. De Biase, G. Impellizzeri, F. Priolo. Phys. Rev. B 73, 195207 (2007).

[17] И.Я. Коренблит, Е.Ф. Шендер. УФН 126, 233 (1978).

[18] В.Ф. Гантмахер. Электроны в неупорядоченных средах. Москва. Физматлит, М. (2013). 288 с.

[19] M. Jamet, A. Barski, T. Devillers, V. Poydenot, R. Dujardin, P. Bayle-Guillemaud, J. Rothman, E. Bellet-Amalric, A. Marty, J. Cibert, R. Mattana, S. Tatarenko. Nature Mater. 5, 653 (2006).

[20] H.H. Woodbury, W.W. Tyler. Phys. Rev. 100, 659 (1955).

[21] R. Rosenbaum. Phys. Rev. B 44, 3599 (1991).

[22] H. Liu, A. Pourret, P. Guyot-Sionnest. ACS Nano 4, 5211 (2010). 